\title{
Fully Developed Forced Convection Heat Transfer in a Porous Channel with Asymmetric Heat Flux Boundary Conditions
}

\author{
Ozgur Cekmer • Moghtada Mobedi • Baris Ozerdem • \\ Ioan Pop
}

Received: 14 November 2010 / Accepted: 22 July 2011 / Published online: 11 August 2011

(C) Springer Science+Business Media B.V. 2011

\begin{abstract}
An analytical study is performed on steady, laminar, and fully developed forced convection heat transfer in a parallel plate channel with asymmetric uniform heat flux boundary conditions. The channel is filled with a saturated porous medium, and the lower and upper walls are subjected to different uniform heat fluxes. The dimensionless form of the DarcyBrinkman momentum equation is solved to determine the dimensionless velocity profile, while the dimensionless energy equation is solved to obtain temperature profile for a hydrodynamically and thermally fully developed flow in the channel. Nusselt numbers for the lower and upper walls and an overall Nusselt number are defined. Analytical expressions for determination of the Nusselt numbers and critical heat flux ratio, at which singularities are observed for individual Nusselt numbers, are obtained. Based on the values of critical heat flux ratio and Darcy number, a diagram is provided to determine the direction of heat transfer between the lower or upper walls while the fluid is flowing in the channel.
\end{abstract}

Keywords Porous media - Internal forced convection · Laminar flow ·

Fully developed flow $\cdot$ Asymmetric boundary condition

\section{List of symbols}

$c_{p} \quad$ Specific heat at constant pressure, $\mathrm{J} / \mathrm{kg} \mathrm{K}$

Da Darcy number

$G \quad$ Pressure gradient in $x$-direction

$H \quad$ Half height of channel, $\mathrm{m}$

\section{O. Cekmer}

Energy Engineering Program, Izmir Institute of Technology, Urla, 35410 Izmir, Turkey

M. Mobedi · B. Ozerdem

Department of Mechanical Engineering, Izmir Institute of Technology, Urla, 35430 Izmir, Turkey

I. Pop (凶)

Faculty of Mathematics, University of Cluj, CP 253, 3400 Cluj, Romania

e-mail: popm.ioan@yahoo.co.uk 
$k \quad$ Thermal conductivity, W/m K

$K \quad$ Permeability, $\mathrm{m}^{2}$

$M \quad$ Viscosity ratio parameter

$\mathrm{Nu} \quad$ Nusselt number

$p \quad$ Pressure, $\mathrm{Pa}$

$q_{1}^{\prime \prime} \quad$ Lower wall subjected heat flux, W/m $\mathrm{m}^{2}$

$q_{\mathrm{u}}^{\prime \prime} \quad$ Upper wall subjected heat flux, $\mathrm{W} / \mathrm{m}^{2}$

$q_{\mu}^{\prime \prime} \quad$ Average wall heat flux, $\mathrm{W} / \mathrm{m}^{2}$

$q_{\mathrm{r}}^{\prime \prime} \quad$ Heat flux ratio

$S \quad$ Porous media shape parameter, $(\mathrm{M} \mathrm{Da})^{-1 / 2}$

$T \quad$ Temperature, $K$

$T_{\mathrm{wl}} \quad$ Lower wall temperature, $\mathrm{K}$

$T_{\text {wu }} \quad$ Upper wall temperature, $\mathrm{K}$

$T_{\mathrm{w} \mu} \quad$ Average wall temperature, $\mathrm{K}$

$u \quad$ Velocity component along the $x$-direction, $\mathrm{m} / \mathrm{s}$

$U \quad$ Dimensionless velocity component along $X$-direction

$\bar{u} \quad$ Average velocity, $\mathrm{m} / \mathrm{s}$

$\bar{U} \quad$ Dimensionless average velocity

$\hat{u} \quad$ Dimensionless normalized velocity

$x \quad$ Coordinate along the axis of the channel, $\mathrm{m}$

$y \quad$ Coordinate normal to the surfaces of the channel, $\mathrm{m}$

$X, Y$ Dimensionless coordinates

\section{Greek letters}

$\beta \quad$ Temperature shape coefficient, Eq. 25

$\mu \quad$ Dynamic viscosity of fluid, $\mathrm{kg} / \mathrm{ms}$

$\mu_{\text {eff }} \quad$ Effective dynamic viscosity, $\mathrm{kg} / \mathrm{ms}$

$\theta \quad$ Dimensionless temperature

$\rho \quad$ Density, $\mathrm{kg} / \mathrm{m}^{3}$

\section{Introduction}

Convective heat transfer in porous media has broad technological applications such as in oil recovery, water supply management in hydrogeology, geothermal exploitation, ground heat storage, building thermal insulation, nuclear waste disposals, radioactive waste management, ground water flow modeling, etc. It is also a subject of interest in environmental and geophysics sciences. A wide applications of the porous media can be found in the well known books by Nield and Bejan (2006), Ingham and Pop (2005), Liu and Masliyah (2005), Vafai (2010), Pop and Ingham (2001), Bejan et al. (2004), and Vadasz (2008), and in the recent article by Harris et al. (2009). They provide significant information on porous media application as well as basic description of heat and fluid flow in porous media.

Literature survey showed that several studies on heat and fluid flow in channels filled with a porous medium have been performed due to importance of the subject. Most of the studies have been performed on channels with symmetrical boundary conditions. For instance, Hooman (2008) investigated analytically the fully developed forced convection flow in a porous medium bounded by two isoflux parallel plates on the basis of Darcy-Brinkman-Forchheimer model. The related momentum and energy equations were solved by an 
asymptotic technique and they found that the value of the Nusselt number is sensitive to the magnitude shape parameter of the porous media, and it is improved with an increase in the value of this parameter. Nield et al. (2004) studied the effects of the viscous dissipation and flow work for a forced convection flow in a channel filled by a saturated porous medium with the walls either held at uniform temperatures or subjected to uniform heat fluxes. The Darcy-Brinkman model was used, and the governing equations were solved analytically. Fully developed flow through a straight porous channel for different flow mechanisms was considered also by Awartani and Hamdan (2005) to study the effects of the porous matrix and the microscopic inertia on the velocity profiles. They obtained velocity profiles for the Darcy-Lapwood-Brinkman and the Darcy-Forchheimer-Brinkman models, and compared the obtained results with the corresponding solutions of the Navier-Stokes flow for Poiseuille, Couette, and Poiseuille-Couette flow regimes. Mobedi et al. (2010) performed an analytical study on the laminar and fully developed forced convection heat transfer in a parallel plate horizontal channel filled with an anisotropic permeability porous medium. The principal axis of the anisotropic porous medium was oriented from 0 to $90^{\circ}$, and a constant heat flux was applied on the outer wall of the channel. Both clear (Newtonian) and Darcy viscous dissipations in the energy equation were considered. They observed that for a particular value of the directional permeability ratio parameter, the external heat applied to the surface of the channel is balanced by the internal heat generation due to viscous dissipation. It is shown that the mean bulk temperature approaches the wall temperature and consequently the Nusselt number becomes infinite. Further studies on heat and fluid flow in porous channels under symmetrical boundary conditions can be found in the literature (Hooman and Ranjbar-Kani 2003, 2004; Tada and Ichimiya 2007; Degan et al. 2002; Kaviany 1985).

A survey of the literature revealed that the number of studies on heat and fluid flow in channels with asymmetric boundary conditions are scarce. For channels and ducts filled with a clear (Newtonian) fluid, the well known book by Shah and London (1978) provides useful information for scientists concerning the asymmetric heating or cooling problems. They investigated the effects of asymmetric boundary conditions for parallel plate channels and circular ducts. In a very good article, Nield (2004) has performed a theoretical study on heat and fluid flow of a viscous (Newtonian) fluid in a parallel plate channel with asymmetric temperature and asymmetric heat flux boundary conditions. He reported that the individual wall Nusselt numbers may not provide sufficient information on heat transfer rate from the channel since the value of the Nusselt number becomes infinite when the heat flux ratio takes the value of 26/9. Hence, Nield (2004) defined an overall Nusselt number based on the average wall heat flux and average wall temperature, respectively. He found that the Nusselt number is independent of the heat flux ratio for the symmetrical flows. He also found that the value of the overall Nusselt number is 140/17 for the Poiseuille flow in a parallel plate channel. Recently, Mitrovic and Maletic (2007) investigated the effect of thermal asymmetric boundary conditions on laminar forced convection heat transfer in a porous plane channel with Darcy dissipation. The walls of the channel were kept at different uniform temperatures. They showed that the thermal asymmetry can lead to a reversal of the heat flux and the corresponding Nusselt number can drop from infinite positive to infinite negative values, or vice versa. The change of the boundary conditions from uniform wall temperature to uniform heat flux leads to a quite different problem (Lin and Wu 1997; Merkin 2009). Thus, the reported results by Mitrovic and Maletic (2007) cannot be used for the present problem concerning boundary condition with asymmetric heat flux.

The problem of forced convection heat transfer in channels and ducts filled with porous media is encountered in many engineering applications, such as hot air flow in crop beds for 
drying or gas flow in adsorbent beds for separation purposes. In the most practical applications, the mechanical power is used to provide the forced convection fluid flow in the channel and the buoyancy effect due to temperature gradient being neglected. The viscous dissipation effect is also neglected for such kind of flows. Pu et al. (1999) performed an experimental study on mixed convection heat transfer in a vertical packed channel with asymmetric heating of opposing walls. They showed that for the asymmetric heated channels with $\mathrm{Ra} / \mathrm{Pe}<1$, the buoyancy effect can be neglected and forced convection becomes the dominant mode of heat transfer.

The aim of this study is to (a) show the effectiveness of the method proposed by Nield (2004) for the channels filled with porous media; (b) discuss the discontinuity in the Nusselt number of each wall and (c) introduce critical heat flux ratio by which heat flux direction between the walls and fluid flowing in the channel can be predicted. Forced convection mode of heat transfer is considered as a dominant mechanism of heat transfer and viscous dissipation is assumed negligible. A special attention is given to the reversal heat flux at boundaries due to different imposed heat fluxes. The Darcy-Brinkman model is used to determine the velocity profiles in the porous channel. This article is the extension studies by Nield (2004, 2008) for a porous parallel plate channel including a detailed discussion on the physical meaning of the obtained results. Thus, it is shown that the overall Nusselt number can be obtained easily based on the average wall temperature and average heat flux imposed at the lower and upper walls, and the individual Nusselt number can then be calculated from the expression of the overall Nusselt number. Based on the explained method, expressions for the overall and individual Nusselt numbers in terms of the shape parameter of the porous media and/or heat flux ratio are obtained. To assure that the obtained results are correct, the dimensionless forms of the Darcy-Brinkman momentum and energy equations are solved both analytically and numerically. To our best of knowledge, the present problem has not been studied before in the aforementioned manner, so that the obtained results are new and original.

\section{The Considered Problem}

A two-dimensional parallel plate channel filled with a porous medium and saturated with an incompressible fluid which is in local thermodynamic equilibrium is considered. The physical model is depicted in Fig. 1, where the Cartesian coordinates $x$ and $y$ are measured along the channel and normal to it, respectively. The channel has a rectangular cross section with height of $2 H$. It is assumed that the uniform heat fluxes, $q_{1}^{\prime \prime}$ and $q_{\mathrm{u}}^{\prime \prime}$, are applied to the channel walls. The heat flux $q_{1}^{\prime \prime}$ has a positive direction, while the direction of $q_{\mathrm{u}}^{\prime \prime}$ is negative. That is why the lower and upper heat fluxes are shown by $q_{1}^{\prime \prime}(+)$ and $q_{\mathrm{u}}^{\prime \prime}(-)$, respectively. The ratio of $q_{1}^{\prime \prime}(+)$ to $q_{\mathrm{u}}^{\prime \prime}(-)$ is called as the heat flux ratio $q_{\mathrm{r}}^{\prime \prime}=q_{1}^{\prime \prime}(+) / q_{\mathrm{u}}^{\prime \prime}(-)$. It is also assumed that the flowing fluid in the channel is Newtonian and its thermophysical properties are assumed to be constant. The porous medium is isotropic with permeability $K$. The flow in the channel is steady, laminar, and hydrodynamically and thermally fully developed.

\section{Governing Equations and Boundary Conditions}

For a hydrodynamically fully developed and unidirectional flow in a parallel plate channel, the following assumptions are valid: 


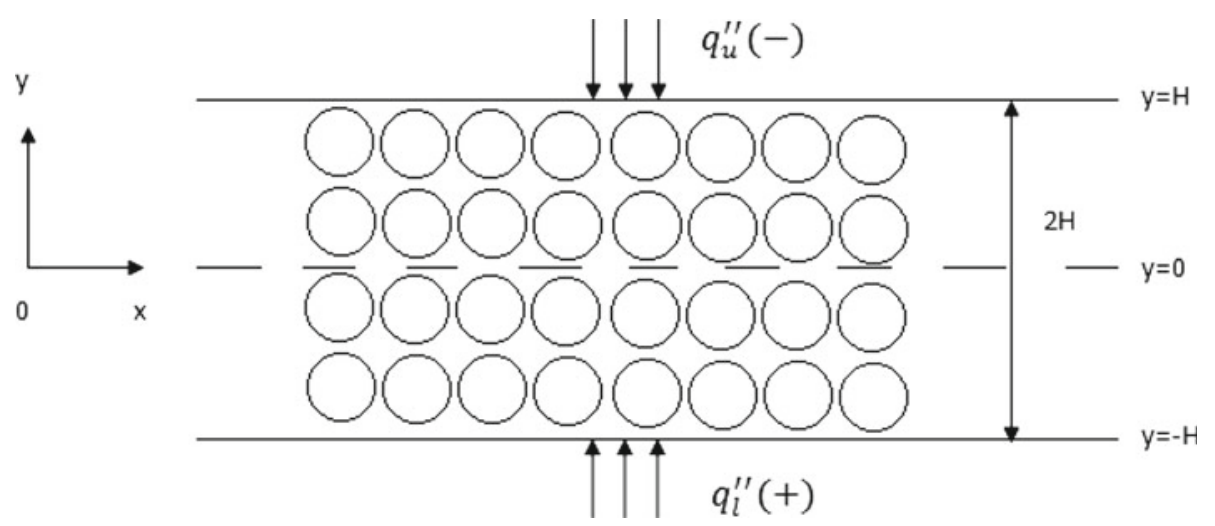

Fig. 1 Physical model of the considered channel filled with an isotropic porous medium

$$
v=0, \quad \frac{\partial u}{\partial x}=0, \quad \frac{\partial p}{\partial y}=0
$$

where $u$ and $v$ are the velocity components in the $x$ - and $y$-directions, and $p$ is the fluid pressure. Because $v$ vanishes for a unidirectional flow in the channel, the Darcy-Brinkman equation for a unidirectional fully developed flow in the channel can be written as:

$$
\mu_{\mathrm{eff}} \frac{\mathrm{d}^{2} u}{\mathrm{~d} y^{2}}-\frac{\mu}{K} u-\frac{\partial p}{\partial x}=0
$$

where $\mu$ represents the dynamic viscosity of the fluid and $\mu_{\text {eff }}$ is the effective viscosity whose value depends on the structure and geometry of the porous medium and of the flow strength (Liu and Masliyah 2005). By considering Fig. 1, the boundary conditions for Eq. 2 can be written as

$$
\begin{aligned}
& \frac{\partial u}{\partial y}=0 \text { at } y=0 \\
& u=0 \text { at } y= \pm H
\end{aligned}
$$

and the mean velocity in the channel can be determined by the following equation:

$$
\bar{u}=\frac{\int_{-H}^{+H} u(y) \mathrm{d} y}{2 H}
$$

To obtain the dimensionless form of the Darcy-Brinkman momentum and energy equations, the following dimensionless parameters are defined:

$$
\begin{gathered}
Y=\frac{y}{H}, \quad X=\frac{x}{H \mathrm{Pe}}, \quad M=\frac{\mu_{\mathrm{eff}}}{\mu}, \quad D a=\frac{K}{H^{2}}, \quad U=\frac{\mu u}{G H^{2}}, \\
G=-\frac{\partial p}{\partial x}, \quad \theta=\frac{T-T_{\mathrm{w} \mu}}{T_{m}-T_{\mathrm{w} \mu}}
\end{gathered}
$$

Substituting parameters of (5) into Eq. 2 yields the following dimensionless form of the Darcy-Brinkman momentum equation:

$$
M \frac{\mathrm{d}^{2} U}{\mathrm{~d} Y^{2}}-\frac{1}{\mathrm{Da}} U+1=0
$$


This equation can be also rewritten in the following form:

$$
\frac{\mathrm{d}^{2} U}{\mathrm{~d} Y^{2}}-S^{2} U+\frac{1}{M}=0
$$

where the coefficient $S$ is called as porous media shape parameter and defined as:

$$
S=\frac{1}{\sqrt{M \mathrm{Da}}}
$$

The dimensionless form of the boundary conditions for Eq. 7 can also be expressed in the following form:

$$
\begin{aligned}
& \frac{\partial U}{\partial Y}=0 \quad \text { at } \quad Y=0 \\
& U=0 \quad \text { at } \quad Y= \pm 1
\end{aligned}
$$

The analytical solution of Eq. 7 with the given boundary conditions (9) yields the dimensionless velocity profile

$$
U(Y)=\mathrm{Da}\left[1-\frac{\cosh (S Y)}{\cosh (S)}\right]
$$

We can also define the dimensionless mean velocity as

$$
\bar{U}=\frac{1}{2} \int_{-1}^{+1} U(Y) \mathrm{d} Y
$$

By using Eqs. 10 and 11, $\bar{U}$ can be calculated as

$$
\bar{U}=\mathrm{Da}\left[1-\frac{\tanh (S)}{S}\right]
$$

Furthermore, the dimensionless velocity profile can be normalized by the following relation:

$$
\hat{u}=\frac{U}{\bar{U}}
$$

Based on the obtained dimensionless velocity given by Eq. 10, and the dimensionless mean velocity of Eq. 12, the dimensionless normalized velocity can be obtained as

$$
\hat{u}=\frac{S \cosh (S)-S \cosh (S Y)}{S \cosh (S)-\sinh (S)}
$$

On the other hand, for a thermally and hydrodynamically fully developed laminar flow in a parallel plate channel, the energy equation can be written as

$$
\rho c_{p} u \frac{\partial T}{\partial x}=k \frac{\partial^{2} T}{\partial y^{2}}
$$

where $\rho$ is the fluid density, $c_{p}$ is the specific heat at constant pressure, and $k$ is the fluid thermal conductivity. Two new variables, namely, the average wall heat flux $q_{\mu}^{\prime \prime}$ and the average wall temperature $T_{\mathrm{w} \mu}$, are defined as

$$
q_{\mu}^{\prime \prime}=\frac{q_{1}^{\prime \prime}+q_{\mathrm{u}}^{\prime \prime}}{2}, \quad T_{\mathrm{w} \mu}=\frac{T_{\mathrm{wu}}+T_{\mathrm{wl}}}{2}
$$


The following equations are valid for the heat fluxes at the lower and upper walls:

$$
q_{1}^{\prime \prime}=h_{1}\left(T_{\mathrm{wl}}-T_{m}\right), \quad q_{\mathrm{u}}^{\prime \prime}=h_{\mathrm{u}}\left(T_{\mathrm{wu}}-T_{m}\right)
$$

Since the heat fluxes, $q_{1}^{\prime \prime}$ and $q_{\mathrm{u}}^{\prime \prime}$, are constant, the differentiation of Eq. 17 with respect to $x$ yields the following relation:

$$
\frac{\mathrm{d}\left(T_{\mathrm{wl}}+T_{\mathrm{wu}}\right) / 2}{\mathrm{~d} x}=\frac{\mathrm{d} T_{\mathrm{w} \mu}}{\mathrm{d} x}=\frac{\mathrm{d} T_{m}}{\mathrm{~d} x}
$$

The energy equation, Eq. 15, can be made dimensionless by using Eq. 5, and the condition of the fully developed forced convection heat transfer in a channel (i.e., $\partial \theta / \partial x=0$ ). Then, the energy equation can be obtained in the following form:

$$
\rho c_{p} u \frac{\mathrm{d} T_{m}}{\mathrm{~d} x}=\frac{k\left(T_{m}-T_{\mu \mathrm{w}}\right)}{H^{2}} \frac{\mathrm{d}^{2} \theta}{\mathrm{d} Y^{2}}
$$

Further, if a control volume with height of $2 \mathrm{H}$ is considered in the channel and the conservation of energy principle is applied, the following relation can be obtained:

$$
\frac{\mathrm{d} T_{m}}{\mathrm{~d} x}=\frac{q_{\mu \mathrm{w}}}{H \rho c_{p} \bar{u}}
$$

By substituting (20) into Eq. 19, the energy equation can be written in the following form:

$$
\frac{u}{\bar{u}} q_{\mu}^{\prime \prime}=\frac{k\left(T_{m}-T_{\mu \mathrm{w}}\right)}{H} \frac{\mathrm{d}^{2} \theta}{\mathrm{d} Y^{2}}
$$

Based on Eq. 16, the average wall heat flux fluxes and the average wall temperatures can be used to define the overall Nusselt number as follows (Nield 2004):

$$
\mathrm{Nu}=\frac{2 H q_{\mu}^{\prime \prime}}{k\left(T_{\mathrm{w} \mu}-T_{m}\right)}
$$

We notice that the average Nusselt number is defined based on the height of the channel. Therefore, instead of two individual Nusselt numbers for the upper and lower walls, a single Nusselt number can be defined. The dimensionless form of the energy equation (15) can be obtained by substituting Eq. 22 into Eq. 21. Thus, we get

$$
\frac{\mathrm{d}^{2} \theta}{\mathrm{d} Y^{2}}+\frac{1}{2} \hat{u} \mathrm{Nu}=0
$$

where the normalized dimensionless velocity $\hat{u}$ is defined by Eq. 13. According to the model of the problem shown in Fig. 1 and by substituting $T_{\mathrm{wl}}$ and $T_{\mathrm{wu}}$ into the dimensionless temperature equation, Eq. 5, the following boundary conditions for the energy equation (23) can be obtained as:

$$
\theta(-1)=\beta, \quad \theta(1)=-\beta
$$

where

$$
\beta=\frac{T_{\mathrm{wl}}-T_{\mathrm{wu}}}{2\left(T_{m}-T_{\mathrm{w} \mu}\right)}
$$

It should be mentioned that the dimensionless temperature profile along channel, including dimensionless wall temperature, is not changed for thermally and hydraulically fully developed flows. The solution of Eq. 23 under the boundary conditions (24) gives the following analytical solution for the dimensionless temperature distribution in the channel: 


$$
\theta(Y)=-\beta Y+\frac{A N u}{2}\left[\frac{\cosh (S)}{2}\left(1-Y^{2}\right)+\frac{\cosh (S Y)-\cosh (S)}{S^{2}}\right]
$$

where

$$
A=\frac{S}{S \cosh (S)-\sinh (S)}
$$

As can be seen from Eq. 26, the dimensionless temperature distribution is a function of the overall Nusselt number $\mathrm{Nu}$, the porous media shape parameter $S$ and the parameter of $\beta$. According to Eq. 25, the value of $\beta$ depends on the surface and mean temperatures, which are unknown. In order to find another expression for $\beta$ in terms of the heat fluxes, further manipulations should be done. The heat fluxes at the walls can be defined as

$$
\begin{aligned}
& q_{1}^{\prime \prime}=-k\left(\frac{\mathrm{d} T}{\mathrm{~d} y}\right)_{y=-H}=-\frac{k}{H}\left(T_{m}-T_{\mathrm{w} \mu}\right)\left(\frac{\mathrm{d} \theta}{\mathrm{d} Y}\right)_{Y=-1} \\
& q_{\mathrm{u}}^{\prime \prime}=-k\left(\frac{\mathrm{d} T}{\mathrm{~d} y}\right)_{y=H}=-\frac{k}{H}\left(T_{m}-T_{\mathrm{w} \mu}\right)\left(\frac{\mathrm{d} \theta}{\mathrm{d} Y}\right)_{Y=1}
\end{aligned}
$$

The differentiation of the dimensionless temperature respect to $Y$ at $Y=-1$ and $Y=1$ can be calculated from the Eq. 26. The ratio of the expressions (28) yields a new definition for $\beta$ in terms of heat flux ratio as:

$$
\beta=\frac{\left(q_{\mathrm{u}}^{\prime \prime}+q_{1}^{\prime \prime}\right)}{\left(q_{\mathrm{u}}^{\prime \prime}-q_{1}^{\prime \prime}\right)} \frac{\mathrm{Nu}}{2}
$$

Here, the compatibility condition

$$
\int_{-1}^{1} \theta \hat{u} \mathrm{~d} Y=2
$$

can be used to obtain an expression for the overall Nusselt number $\mathrm{Nu}$. Thus, $\mathrm{Nu}$ can be obtained by substituting (14) and (26) into (30). Thus, the following expression can be obtained for $\mathrm{Nu}$ after the integration procedure:

$$
\mathrm{Nu}=\frac{24 S[S \cosh (S)-\sinh (S)]^{2}}{15 \sinh (2 S)+\left(4 S^{3}-24 S\right) \cosh ^{2}(S)-6 S}
$$

As it was mentioned before, it is possible to obtain expressions for individual Nu numbers $\left(\mathrm{Nu}_{1}\right.$ and $\left.\mathrm{Nu}_{\mathrm{u}}\right)$ using the expression (31) for $\mathrm{Nu}$. The individual $\mathrm{Nu}$ numbers for the lower and upper plates are defined as:

$$
\mathrm{Nu}_{1}=\frac{2 H q_{1}^{\prime \prime}}{k\left(T_{\mathrm{wl}}-T_{m}\right)}, \quad \mathrm{Nu}_{\mathrm{u}}=\frac{2 H q_{\mathrm{u}}^{\prime \prime}}{k\left(T_{\mathrm{wu}}-T_{m}\right)}
$$

Further, the energy conservation principle can be applied at each wall of the channel and the following expressions can be obtained:

$$
\begin{aligned}
h_{\mathrm{l}}\left(T_{m}-T_{\mathrm{wl}}\right) & =\frac{k}{H}\left(T_{m}-T_{\mathrm{w} \mu}\right)\left(\frac{\partial \theta}{\partial Y}\right)_{Y=-1}, \\
h_{\mathrm{u}}\left(T_{m}-T_{\mathrm{wu}}\right) & =\frac{k}{H}\left(T_{m}-T_{\mathrm{w} \mu}\right)\left(\frac{\partial \theta}{\partial Y}\right)_{Y=1}
\end{aligned}
$$


Fig. 2 Possible signs of the Nusselt numbers at lower and upper plates
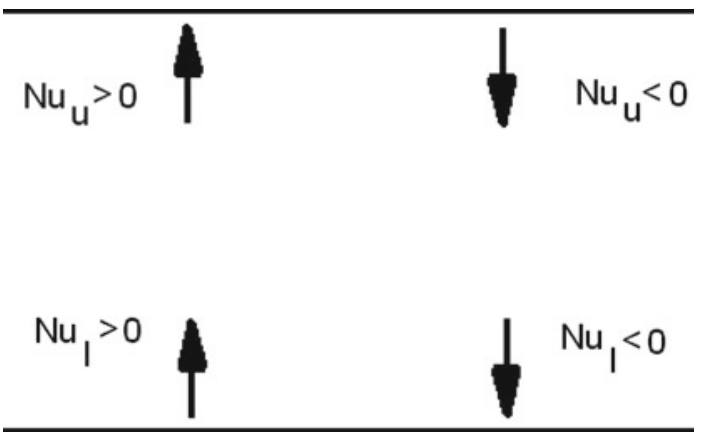

After determination of the dimensionless temperature gradient at the walls using Eq. 26 and performing some mathematical manipulations, the individual Nu numbers can be determined from Eq. 33 as:

$$
\mathrm{Nu}_{\mathrm{l}}=\frac{4 \mathrm{Nu}}{(\mathrm{Nu}+2)+(\mathrm{Nu}-2) / q_{\mathrm{r}}^{\prime \prime}}, \quad \mathrm{Nu}_{\mathrm{u}}=\frac{-4 \mathrm{Nu}}{(\mathrm{Nu}+2)+(\mathrm{Nu}-2) q_{\mathrm{r}}^{\prime \prime}}
$$

where $q_{\mathrm{r}}^{\prime \prime}$ is the heat fluxes ratio (i.e., $q_{\mathrm{r}}^{\prime \prime}=q_{1}^{\prime \prime} / q_{\mathrm{u}}^{\prime \prime}$ ). The overall Nu number can be calculated from Eq. 31 for a given porous channel and by substituting the Nusselt number and heat flux ratio into Eq. 34, the expressions for the individual $\mathrm{Nu}$ numbers can be obtained. Figure 2 shows the sign of the Nusselt number according to the direction of the heat fluxes between the walls of the channel. For example, the positive value of $\mathrm{Nu}$ number at the lower plates indicates the heat transfer from the wall to the porous media, while the negative value of $\mathrm{Nu}$ number refers to the heat transfer from the fluid to the lower plate. The denominators of Eq. 34 take zero value for the specific values of the heat fluxes ratios defined as

$$
q_{\mathrm{rl}, \mathrm{cr}}^{\prime \prime}=\frac{2-\mathrm{Nu}}{2+\mathrm{Nu}}, \quad q_{\mathrm{ru}, \mathrm{cr}}^{\prime \prime}=\frac{2+\mathrm{Nu}}{2-\mathrm{Nu}}
$$

where $q_{\mathrm{rl}, \mathrm{cr}}^{\prime \prime}$ and $q_{\mathrm{ru}, \mathrm{cr}}^{\prime \prime}$ represent the critical heat flux ratios for the lower and the upper plates, respectively. Singularities in the individual $\mathrm{Nu}$ numbers exist at these critical heat flux ratios. The values of $\mathrm{Nu}$ numbers at the lower and upper plate approach to infinity and the heat transfer at the lower and upper plates changes its direction at $q_{\mathrm{rl}, \mathrm{cr}}^{\prime \prime}$ and $q_{\mathrm{ru}, \mathrm{cr}}^{\prime \prime}$.

\section{Numerical Analysis}

As it was mentioned in the preceding section, the dimensionless governing equations for hydrodynamically and thermally fully developed flow in a porous channel are the DarcyBrinkman momentum equation and the energy equations, namely, Eqs. 7 and 23. We will present further, numerical results for these equations. The Darcy-Brinkman Eq. 7 along with the boundary condition (9) is solved by a finite-difference method. The following transformation is used for the compatibility condition:

$$
\Psi=\frac{\theta}{\mathrm{Nu}}
$$

Since the overall Nusselt number is constant for the fully developed flow in the channel, a new form of the dimensionless energy equation (23) and boundary conditions based on the $\Psi$ function can be obtained as: 


$$
\frac{\mathrm{d}^{2} \Psi}{\mathrm{d} Y^{2}}+\frac{1}{2} \hat{u}=0
$$

subject to the boundary conditions

$$
\Psi(-1)=\beta / \mathrm{Nu}, \quad \Psi(1)=-\beta / \mathrm{Nu}
$$

Therefore, the new form of the compatibility condition is given now by

$$
\int_{-1}^{1} \Psi \hat{u} \mathrm{~d} Y=2 / \mathrm{Nu}
$$

We notice that the boundary conditions (38) depend on the overall Nusselt number, which is unknown yet. The distribution of $\Psi$ is found according to an initial guess value for the overall Nusselt number. Then, a new value for $\mathrm{Nu}$ number is found using the compatibility condition (39). This new value of the overall Nusselt number was used to obtain the new distribution of $\Psi$ and consequently the new value for overall Nusselt number is obtained. The procedure is continued until the absolute difference between the new and old calculated Nusselt numbers becomes less than $10^{-9}$. The number of nodes in the $Y$-direction is 200 in the performed numerical study.

\section{Results and Discussion}

The overall and individual Nusselt numbers are calculated and their changes with the Darcy number and heat flux ratio are discussed via graphics and tables. In order to easily realize the physical meaning of the obtained analytical solutions, the value of the viscosity ratio is assumed as 1 (i.e., $M=1$ ) and the discussion is performed based on the Darcy number. As it was mentioned before, the energy equation is solved both numerically and analytically to ensure that the obtained results are correct. Table 1 shows the overall and individual $\mathrm{Nu}$ numbers calculated both analytically and numerically for a channel with $\mathrm{Da}=0.01$ and for four different values of the heat flux ratio $q_{\mathrm{r}}^{\prime \prime}$ as 1,2,10, and 100. As can be seen from Table 1 , there is a good agreement between the numerical and analytical solutions. Table 2 is also prepared to indicate the accuracy of the obtained results. In this table, the overall and individual Nusselt numbers for two channls (one is a channel filled with a porous medium with $\mathrm{Da}=1000$ and for another channel filled with a clear fluid) are presented for different values of the heat flux ratios, $q_{\mathrm{r}}^{\prime \prime}$. Table 2 shows that the overall and individual Nusselt numbers are almost identical for the two considered channels.

Figure 3 shows the variation of the overall Nusselt number with Darcy number. As can be seen from Eq. 31, the overall Nusselt number does not depend on the heat flux ratio. For a specified Darcy number, the value of the overall Nusselt number is constant. No singularity is observed in the variation of the overall Nusselt with Darcy number. Hence, it can be calculated the heat transfer rate to/from the parallel plates for the entire possible values of heat flux ratio and Darcy number. The total heat transfer rate for different channels can be easily calculated and compared with each others if the definition of the overall Nusselt number is considered. Figure 3 indicates that the overall Nusselt number steeply drops with the increase of Darcy number in the region of $\mathrm{Da}<1$. The value of the overall Nusselt number becomes almost constant for high values of the Darcy number (i.e., Da $\gg 1$ ). For high values of Darcy number (i.e., fluid behaves like a clear fluid) the overall Nusselt number takes value of 4.11 
Table 1 Comparison of the analytical and numerical results for the Nusselt numbers of a flow in a channel filled with a porous medium when $\mathrm{Da}=0.01$

\begin{tabular}{|c|c|c|c|c|c|c|}
\hline & \multicolumn{3}{|c|}{ Nusselt number from Analytical solution } & \multicolumn{3}{|c|}{ Nusselt number from numerical solution } \\
\hline & $\mathrm{Nu}_{1}$ & $\mathrm{Nu}_{\mathrm{u}}$ & $\mathrm{Nu}$ & $\mathrm{Nu}_{1}$ & $\mathrm{Nu}_{\mathrm{u}}$ & $\mathrm{Nu}$ \\
\hline$q_{1}^{\prime \prime} / q_{\mathrm{u}}^{\prime \prime}=1$ & 5.1293 & -5.1293 & 5.1293 & 5.1281 & -5.1281 & 5.1281 \\
\hline$q_{1}^{\prime \prime} / q_{\mathrm{u}}^{\prime \prime}=2$ & 3.6871 & -23.564 & 5.1293 & 3.6866 & -23.524 & 5.1281 \\
\hline$q_{1}^{\prime \prime} / q_{\mathrm{u}}^{\prime \prime}=10$ & 3.0100 & 0.8491 & 5.1293 & 3.0097 & 0.8493 & 5.1281 \\
\hline$q_{1}^{\prime \prime} / q_{\mathrm{u}}^{\prime \prime}=100$ & 2.8906 & 0.0671 & 5.1293 & 2.8904 & 0.0671 & 5.1281 \\
\hline
\end{tabular}

Table 2 Comparison of the variation of the Nusselt number for a channel filled with a clear (viscous) fluid and also with a porous medium for $\mathrm{Da}=1000$

\begin{tabular}{llllllll}
\hline & \multicolumn{3}{l}{ Nusselt number for $\mathrm{Da}=1000$} & & \multicolumn{3}{l}{ Nusselt number for a clear fluid } \\
\cline { 2 - 3 } & $\mathrm{Nu}_{1}$ & $\mathrm{Nuu}$ & $\mathrm{Nu}$ & & $\mathrm{Nu}$ & $\mathrm{Nuu}$ & $\mathrm{Nu}$ \\
\hline$q_{1}^{\prime \prime} / q_{\mathrm{u}}^{\prime \prime}=1$ & 4.1177 & -4.1177 & 4.1177 & & 4.1177 & -4.1177 & 4.1177 \\
$q_{1}^{\prime \prime} / q_{\mathrm{u}}^{\prime \prime}=2$ & 3.2558 & -8.7503 & 4.1177 & & 3.2558 & -8.7500 & 4.1177 \\
$q_{1}^{\prime \prime} / q_{\mathrm{u}}^{\prime \prime}=10$ & 2.7889 & 1.0937 & 4.1177 & & 2.7888 & 1.0937 & 4.1177 \\
$q_{1}^{\prime \prime} / q_{\mathrm{u}}^{\prime \prime}=100$ & 2.7017 & 0.0801 & 4.1177 & & 2.7017 & 0.0801 & 4.1177 \\
\hline
\end{tabular}

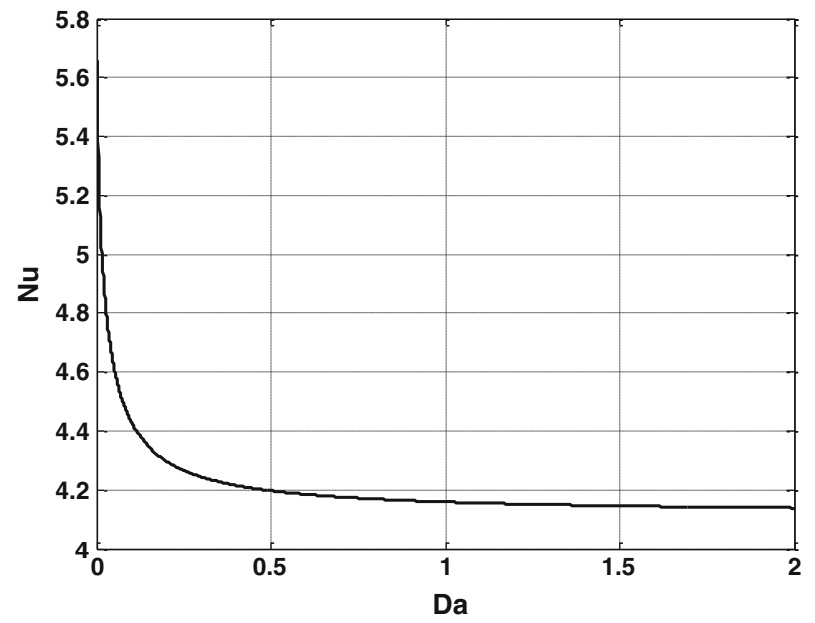

Fig. 3 Changes in the overall Nusselt number with the Darcy number

which is identical to the value of the Nusselt number for a fully developed flow in a channel filled with a clear (viscous) fluid of height $2 H$ (Nield 2004).

The variations of the Nusselt numbers $\mathrm{Nu}_{l}$ and $\mathrm{Nu}_{\mathrm{u}}$ at the lower and upper plates of the channel with the heat flux ratio $q_{\mathrm{r}}^{\prime \prime}$ are illustrated in Fig. $4 \mathrm{a}$ and b, respectively. These figures were prepared for a flow in a channel with $\mathrm{Da}=100$, to first discuss the heat and fluid flow in a channel filled with a clear fluid and with asymmetry heat flux boundary conditions. As 
Fig. 4 Variation of the individual Nusselt number with absolute value of heat flux ratio when $\mathrm{Da}=100$ : a lower plate; b upper plate
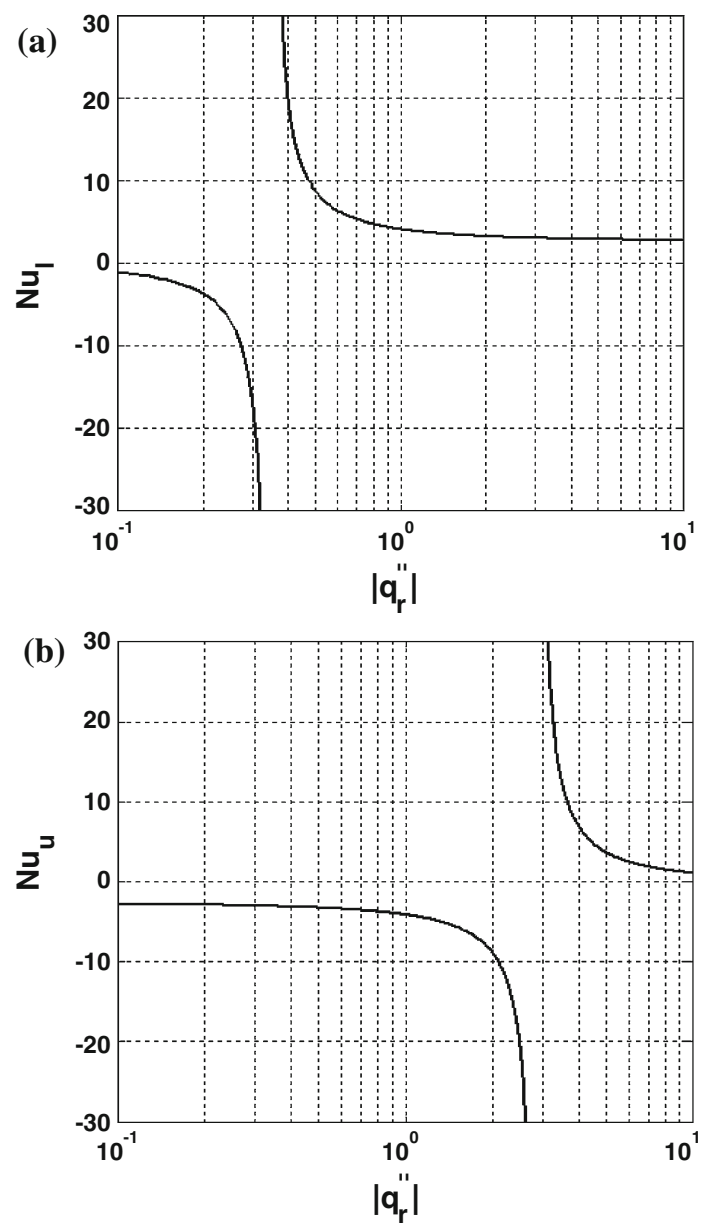

can be observed from Fig. 4a, the value of $\mathrm{Nu}_{l}$ is almost zero for low values of the heat flux ratio $q_{\mathrm{r}}^{\prime \prime}$ such as, for example, $q_{\mathrm{r}}^{\prime \prime}=10^{-1}$. Thus, for low values of $q_{\mathrm{r}}^{\prime \prime}$, the value of $\mathrm{Nu}_{l}$ is negative and it refers to the fact that the heat transfer takes place from the fluid to the lower plate. For values of $q_{\mathrm{r}}^{\prime \prime}$ greater than $q_{\mathrm{r}}^{\prime \prime}=10^{-1}$, the values of $\mathrm{Nu}_{l}$ decrease with increase in $q_{\mathrm{r}}^{\prime \prime}$. This trend continues up to a heat flux ratio of $q_{\mathrm{r}}^{\prime \prime}=0.346$. The value of $\mathrm{Nu}_{l}$ is changed at $q_{\mathrm{r}}^{\prime \prime}=0.346$ and it then takes positive values at the lower plate of the channel. A singularity value of the Nusselt number at the lower plate, $\mathrm{Nu}_{l}$, is observed at $q_{\mathrm{r}}^{\prime \prime}=0.346$. In this study, the heat flux ratio $q_{\mathrm{r}}^{\prime \prime}$ at which the heat flux between wall and fluid changes its direction is called a critical heat flux ratio. It found also that in the channels with $\mathrm{Da}=$ $100, \mathrm{Nu}_{l}$ at the lower plate takes positive value in the region of $q_{\mathrm{r}}^{\prime \prime}>q_{\mathrm{rl}, \mathrm{cr}}^{\prime \prime}$, and the value of $\mathrm{Nu}_{l}$ decreases with the further increase of the heat flux ratio in the region of $q_{\mathrm{r}}^{\prime \prime}>q_{\mathrm{rl}, \mathrm{cr}}^{\prime \prime}$. The value of $\mathrm{Nu}_{l}$ approaches to 2.789 for high values of the heat flux ratio $q_{\mathrm{r}}^{\prime \prime}$, such as $q_{\mathrm{r}}^{\prime \prime}=10$. On the other hand, the variation of the Nusselt number at the upper plate, $\mathrm{Nu}_{\mathrm{u}}$, with the heat flux ratio $q_{\mathrm{r}}^{\prime \prime}$ is shown in Fig. $4 \mathrm{~b}$ when $\mathrm{Da}=100$. It is noticed that for low values of the heat flux ratio $q_{\mathrm{r}}^{\prime \prime}$, such as $q_{\mathrm{r}}^{\prime \prime}=10^{-1}$, the value of $\mathrm{Nu}_{\mathrm{u}}$ is almost constant and equals to 
$\mathrm{Nu}_{\mathrm{u}}=-2.789$. It is also observed that the value of $\mathrm{Nu}_{\mathrm{u}}$ is negative, which indicates that the heat transfer takes place from the upper plate to the fluid. It is further noticed that the value of $\mathrm{Nu}_{\mathrm{u}}$ decreases with the increase of the heat flux ratio $q_{\mathrm{r}}^{\prime \prime}$, and at a critical value of $q_{\mathrm{r}}^{\prime \prime}$, namely, $q_{\mathrm{ru}, \mathrm{cr}}^{\prime \prime}=2.889, \mathrm{Nu}_{\mathrm{u}}$ changes its direction so that the Nusselt number at the upper plate, $\mathrm{Nu}_{l}$, takes positive values. This change of the heat flux direction refers to the heat transfer from the fluid to the upper plate. For the regions with $q_{\mathrm{ru}, \mathrm{cr}}^{\prime \prime}>2.889$, further increase in heat flux ratio $q_{\mathrm{r}}^{\prime \prime}$ causes $\mathrm{Nu}_{\mathrm{u}}$ approaches to zero so that the upper plate behaves like an insulated wall. In fact, Fig. 4 shows that three different heat flux regions should be considered in analyzing of the heat transfer in a porous channel with asymmetric boundary conditions. The first region is the region with $q_{\mathrm{r}}^{\prime \prime}>q_{\mathrm{rl}, \mathrm{cr}}^{\prime \prime}$, for which the value of the Nusselt number at the lower plate, $\mathrm{Nu}_{l}$, is negative and heat is transferred from the fluid to the lower plate. In this region, the value of the Nusselt number at the upper plate, $\mathrm{Nu}_{\mathrm{u}}$, is also negative and it refers to the heat transfer from the upper plate to the porous medium. The second region is the region in which $q_{\mathrm{rl}, \mathrm{cr}}^{\prime \prime}<q_{\mathrm{r}}^{\prime \prime}<q_{\mathrm{ru}, \mathrm{cr}}^{\prime \prime}$. In this region, the values of $\mathrm{Nu}_{l}$ are positive, while $\mathrm{Nu}_{\mathrm{u}}$ takes negative values. Heat is transferred here from the both upper and lower plates to the porous media. The third region is characterized by $q_{\mathrm{r}}^{\prime \prime}>q_{\mathrm{ru}, \mathrm{cr}}^{\prime \prime}$ for which the values of both $\mathrm{Nu}_{l}$ and $\mathrm{Nu}_{\mathrm{u}}$ are positive. This shows that heat is transferred from the lower plate to the fluid, while the upper plate receives heat from the fluid flowing through the channel.

Figure 5 shows the changes of the individual Nusselt numbers with the heat flux ratio $q_{\mathrm{r}}^{\prime \prime}$ for $\mathrm{Da}=0.1,0.01$, and 0.001. As can be observed, the changes of $\mathrm{Nu}_{l}$ for different values of the Darcy numbers are very similar to the variation of $\mathrm{Nu}_{l}$ with $\mathrm{Da}=100$. This feature reveals that the value of critical heat flux ratio $q_{\mathrm{r}}^{\prime \prime}$ is not constant for a porous channel and it varies with the Darcy number. By decrease of the Darcy number, the critical value of the heat flux ratio at the lower plate slightly increases, while a small decrease of the critical heat flux ratio at the upper plate is observed.

Finally, Fig. 6 indicates the variation of the critical heat flux ratio at the lower and upper plates with the Darcy numbers between $10^{-5}$ and $10^{2}$. The value of the critical heat flux at the lower plate is about 0.4 , while the value of the critical heat flux at the upper plate is about 2.5. As can be observed from Fig. 6, the heat transfer between the upper and lower plates with the fluid flowing in the channel can be divided into three regions. Once the value of the Darcy number (or $S$ parameter) is known for a channel filled with porous media, the critical heat flux ratio for the lower and upper plates can easily be found by using Eq. 35. The direction of the heat transfer between the plates and fluid flowing in the channel can be predicted by comparing the obtained critical heat flux ratio at the upper and lower plates and the subjected critical heat flux ratio to the channel. For example, for a porous channel with $\mathrm{Da}=10^{-4}$ and $M=1$, the critical heat flux ratio for the lower plate is $q_{\mathrm{rl}, \mathrm{cr}}^{\prime \prime}=0.492$ and for the upper plate it is $q_{\mathrm{ru}, \mathrm{cr}}^{\prime \prime}=2.029$. Hence, for the same channel with $q_{\mathrm{r}}^{\prime \prime}=0.1$, it is easy to predict that heat is transferred from the upper plate to the fluid and also from fluid to the lower plate since $q_{\mathrm{r}}^{\prime \prime}<q_{\mathrm{rl}, \mathrm{cr}}^{\prime \prime}$ and $q_{\mathrm{r}}^{\prime \prime}<q_{\mathrm{ru}, \mathrm{cr}}^{\prime \prime}$, respectively. For the same channel but with $q_{\mathrm{r}}^{\prime \prime}=1$, the direction of the heat transfer at the lower plate is changed. For this channel, heat is transferred from the lower plate to the fluid since $q_{\mathrm{r}}^{\prime \prime}>q_{\mathrm{rl}, \mathrm{cr}}^{\prime \prime}$ and the transfer of heat from upper plate to the fluid continues until the condition $q_{\mathrm{r}}^{\prime \prime}<q_{\mathrm{ru}, \mathrm{cr}}^{\prime \prime}$ is realized. Finally, for the same porous channel with $q_{\mathrm{r}}^{\prime \prime}=5$, the direction of heat transfer at the upper plate is changed and heat is transferred from the fluid to the upper plate because of the condition $q_{\mathrm{r}}^{\prime \prime}>q_{\mathrm{ru}, \mathrm{cr}}^{\prime \prime}$. The transfer of heat from the lower plate to the channel continues until the condition $q_{\mathrm{r}}^{\prime \prime}<q_{\mathrm{rl}, \mathrm{cr}}^{\prime \prime}$ is realized. It should be mentioned that the critical heat flux ratio is almost constant for very small values of the Darcy number $(\mathrm{Da}<0.001)$ or large values of the Darcy number $(\mathrm{Da}>10)$ as can be observed in Fig. 6. 
Fig. 5 Variation of the individual Nusselt number with absolute value of the heat flux ratio when $\mathrm{Da}=0.1,0.01$, and 0.001: a lower plate; $\mathbf{b}$ upper plate
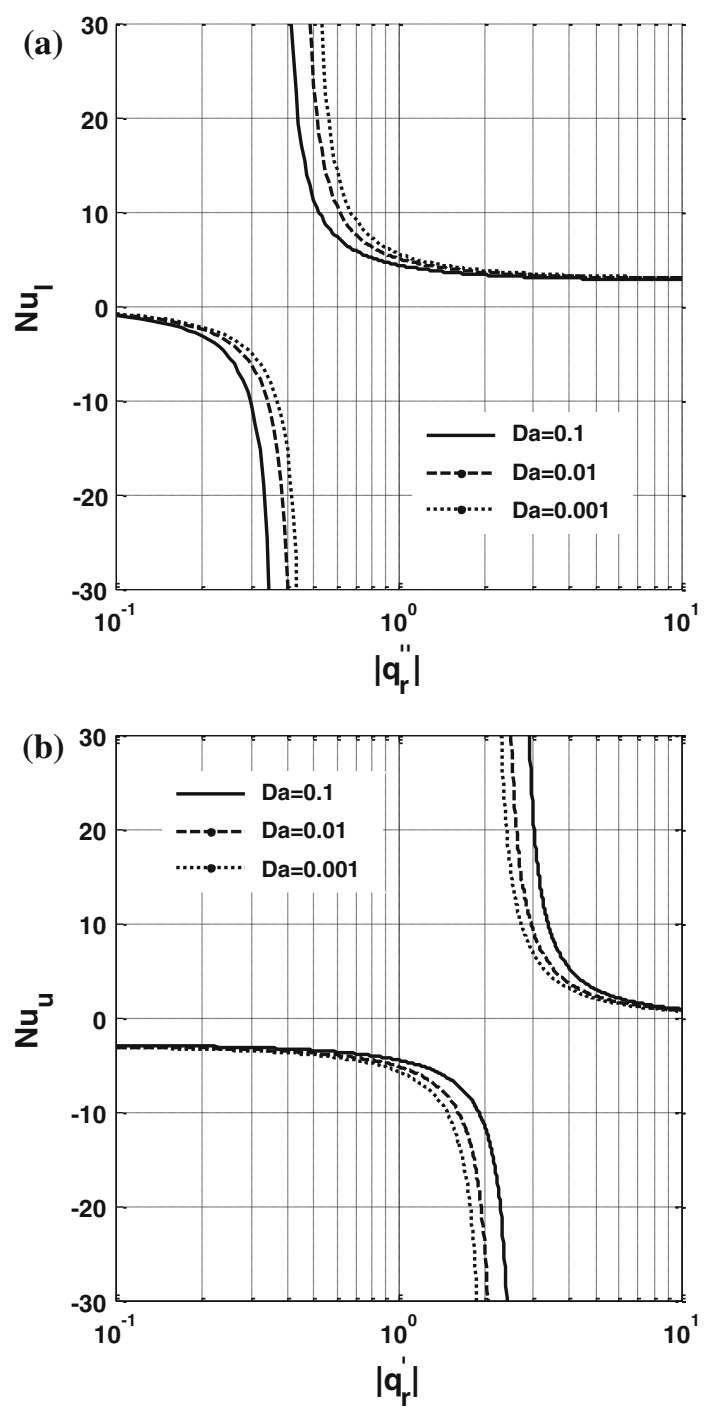

\section{Conclusion}

The steady fully developed flow and heat transfer in a channel filled with a porous medium and with asymmetric boundary conditions are analyzed. Based on the ratio of heat fluxes applied at the walls, analytical expressions are determined for the individual and overall Nusselt numbers. Using the overall Nusselt number, defined based on the average walls heat fluxes and average wall temperatures, can determine the total heat transfer rate between the plates and the flowing fluid. It is found that the variation of the overall Nusselt number is continuous for all considered values of the Darcy number, and it does not depend on the heat flux ratio. However, it does not provide any information about the direction of the heat flux between the plates and fluid. Hence, expressions for the individual Nusselt numbers are also necessary in practice. 


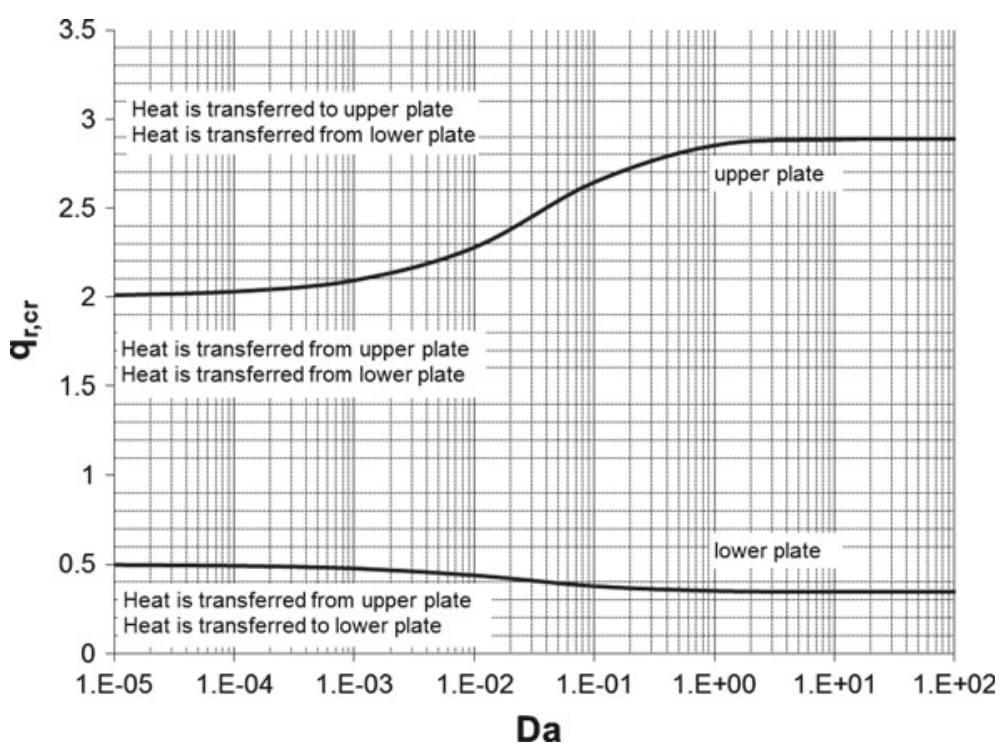

Fig. 6 Change in critical heat flux with Darcy number

Critical values of the heat flux ratio, at which the heat transfer changes its direction at the plate, is defined and determined in this study. It is shown that the critical heat flux ratio for each plate is a function of the Darcy number when the dynamic viscosity ratio is $1(M=1)$. The obtained results showed that three different regions can be defined according to the lower and upper critical heat flux ratios. The directions of the heat fluxes at the lower and upper plates are different in these three regions. For a porous channel with specified heat flux ratio and Darcy number, the direction of heat transfer at the lower and upper plates with fluid flowing in the porous media can be predicted by comparing the imposed heat flux ratio and the critical heat flux ratio for the each plate.

\section{References}

Awartani, M.M., Hamdan, M.H.: Fully developed flow through a porous channel bounded by flat plates. Appl. Math. Comput. 169, 749-757 (2005)

Bejan, A., Dincer, I., Lorente, S., Miguel, A.F., Reis, A.F.: Porous and Complex Flow Structures in Modern Technologies. Springer, New York (2004)

Degan, D., Zohoun, S., Vasseur, P.: Forced convection in horizontal porous channels with hydrodynamic anisotropy. Int. J. Heat Mass Transf. 45, 3181-3188 (2002)

Harris, S.D., Ingham, D.B., Pop, I.: Mixed convection boundary-layer flow near the stagnation point on a vertical surface in a porous medium: Brinkman model with slip. Transp. Porous Media 77, 267-285 (2009)

Hooman, K.: A perturbation solution for forced convection in porous-saturated duct. J. Comput. Appl. Math. 211, 57-66 (2008)

Hooman, K., Ranjbar-Kani, A.A.: Forced convection in a fluid-saturated porous-medium tube with isoflux wall. Int. Commun. Heat Mass Transf. 30, 1015-1026 (2003)

Hooman, K., Ranjbar-Kani, A.A.: A perturbation based analysis to investigate forced convection in a porous saturated tube. J. Comput. Appl. Math. 162, 411-419 (2004)

Ingham, D.B., Pop, I. (eds.): Transport Phenomena in Porous Media. Elsevier, Oxford (2005)

Kaviany, M.: Laminar flow through a porous channel bounded by isothermal parallel plates. Int. J. Heat Mass Transf. 28, 851-858 (1985) 
Lin, H.-T., Wu, C.-M.: Combined heat and mass transfer by laminar natural convection from a vertical plate with uniform heat flux and concentration. Heat Mass Transf. 32, 293-299 (1997)

Liu, S., Masliyah, J.H.: In: Vafai, K. (ed.) Handbook of Porous Media (2nd edition), pp. 111. Taylor \& Francis, New York (2005)

Merkin, J.H.: Natural convective boundary-layer flow in a heat generating porous medium with a prescribed wall heat flux. J. Appl. Math. Phys. (ZAMP) 60, 543-564 (2009)

Mitrovic, J., Maletic, B.: Heat Transfer with laminar forced convection in a porous channel exposed to a thermal asymmetry. Int. J. Heat Mass Transf. 50, 1106-1121 (2007)

Mobedi, M., Cekmer, O., Pop, I.: Forced convection heat transfer inside an anisotropic porous channel with oblique principal axes: effect of viscous dissipation. Int. J. Therm. Sci. 49, 1984-1993 (2010)

Nield, D.A.: Forced convection in a parallel plate channel with asymmetric heating. Int. J. Heat Mass Transf. 47, 5609-5612 (2004)

Nield, D.A.: Erratum to "Forced convection in a parallel plate channel with asymmetric heating". Int. J. Heat Mass Transf. 51, 2108 (2008)

Nield, D.A., Bejan, A.: Convection in Porous Media. Springer, New York (2006)

Nield, D.A., Kuznetsov, A.V., Xiong, M.: Effects of viscous dissipation and flow work on forced convection in a channel filled by a saturated porous medium. Transp. Porous Media 56, 351-367 (2004)

Pop, I., Ingham, D.B.: Convective Heat Transfer: Mathematical and Computational Modelling of Viscous Fluids and Porous Media. Pergamon, Oxford (2001)

Pu, W.L., Cheng, P., Zhao, T.S.: An experimental study of mixed convection heat transfer in vertical packed channels. J. Thermo Phys. Heat Transf. 13, 517-521 (1999)

Shah, R.K., London, A.L.: Advances in Heat Transfer, Suppl 1. Academic Press, New York (1978)

Tada, S., Ichimiya, K.: Analysis of laminar dissipative flow and heat transfer in a porous saturated circular tube with constant wall heat flux. Int. J. Heat Mass Transf. 50, 2406-2413 (2007)

Vadasz, P. (ed.): Emerging Topics in Heat and Mass Transfer in Porous Media. Springer, New York (2008)

Vafai, K.: Porous Media: Applications in Biological Systems and Biotechnology. CRC Press, Tokyo (2010) 